\title{
An Improved Mobility Aware Relaying Scheme for Body Area Networks
}

This paper was downloaded from TechRxiv (https://www.techrxiv.org).

\section{LICENSE}

CC BY 4.0

SUBMISSION DATE / POSTED DATE

$17-04-2020 / 17-04-2020$

\section{CITATION}

Michaelides, Costas; lloridou, Maria; Pavlidou, Foteini-Niovi (2020): An Improved Mobility Aware Relaying Scheme for Body Area Networks. TechRxiv. Preprint. https://doi.org/10.36227/techrxiv.12141012.v1

$\mathrm{DOI}$

10.36227/techrxiv.12141012.v1 


\title{
An Improved Mobility Aware Relaying Scheme for Body Area Networks
}

\author{
Costas Michaelides, Maria Iloridou and Fotini-Niovi Pavlidou
}

\begin{abstract}
Communication in Body Area Networks (BANs) involves weak signals, due to safety regulations, huge pathloss from the absorption and usually high mobility. In this work, we introduce an improved mobility aware relaying scheme for BANs, as an alternative to the two-hop star topology extension of IEEE 802.15.6-2012, in order to enhance packet delivery. Specifically, an emergency phase (EP) is added after the regular random access phase (RAP1) of the superframe and the connected nodes transmit rescue beacons to reach disconnected nodes. When a disconnected node receives a rescue beacon, it participates in the current EP. The packets are buffered and relayed to the hub by the connected nodes. Simulation results show that it is feasible to receive more packets compared to the standard with a justified increase of energy consumption due to random access which is compensated with increased packet delivery.
\end{abstract}

Index Terms-Body Area Networks, MAC, Relaying

\section{INTRODUCTION}

Body Area Networks (BANs) are considered today as one of the most promising approaches for improving the quality of human life. Through real-time interactive health applications, it is possible to develop remote patient monitoring systems, in order to meet the every day needs of the aging population and to carry out diagnostic and curative programs for a variety of diseases. BANs consist of a number of portable, miniaturized, and autonomously interconnected sensor nodes, which are located either inside or outside of the human body. Thus, they are special sensor networks and the main body of the literature on wireless sensor networks can be utilized to study them.

During the last two decades significant research was carried out on hardware and software development, network design, communication protocols and physical channel implications, by taking into account the biological characteristics of the human body. Several factors affect the reliability of BAN applications, such as energy consumption, residual energy, network capacity, interference, data aggregation, pathloss, traffic characteristics, and, mostly the network connectivity. The latter is of main importance, due to the movement of the human body, which results to frequent topology changes. All these issues are included, after theoretical and empirical investigations, in a standard specially addressing the BAN applications, the IEEE 802.15.6-2012 [1]. This was released back in 2012 and supports a variety of data rates for the communication of nodes in one-hop star or two-hop extended star topology on, in or close to the human body.

Currently, the research in BANs is focused on energy efficiency (EE) and quality of service (QoS). Also, the deploy-

Costas Michaelides (cmich@auth.gr), Maria Iloridou (iloridou@auth.gr) and Fotini-Niovi Pavlidou (niovi@auth.gr) are with School of Electrical and Computer Engineering, Aristotle University of Thessaloniki, Greece.

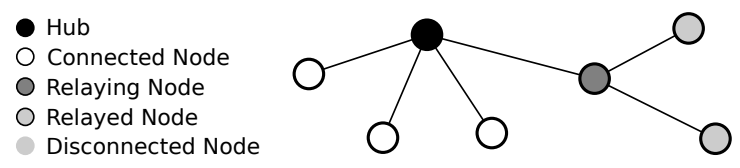

Figure 1. Two-hop extended star topology of IEEE 802.15.6-2012

ment of network applications requires the investigation of programmability [2]. In general, there is need for simple solutions to avoid complex transceivers and keep energy consumption at low levels. The first choice regarding communication is star topology, because the distances are too short. However, the signals are weak and human body is constantly in motion, so it is very common for a node to draw away and disconnect from the hub. Connectivity issues may be addressed decentralized as well, as in [3]. In this work, we investigate the relaying process instead of more complex structures, to preserve the efficiency of the standard.

\section{A. Related literature}

Relaying as a store and forward scheme, is already supported by the IEEE 802.15.6-2012. This standard provides a connection method and scheduled access for the relayed nodes through a two-hop extended star topology, as in Fig. 1. However, this function is not included in the current implementation of the ETSI SmartBAN [4].

During the last decade, several works employed relaying as it is a feasible way to address connectivity in practice. Earlier in 2007, [5] introduced a cross-layer protocol for MAC and routing. This protocol sets up a tree structured network to route the data packets to the sink without collisions. BATMAC [6], also an early work, introduces a TDMA MAC with relay support. A protocol based on BATMAC in [7], manages the tradeoff between QoS and energy consumption. The multi-hop protocol in [8] uses a gossiping strategy between neighbors and adapts transmit power to optimize power consumption. The authors extended their work in [9], while in [10] was suggested an alternative approach to simplify the two-hop topology extension of the standard. In this case, relaying is implemented only during the uplink and thus, the packets from the hub are transmitted directly to relayed nodes. In [11], a topology design was introduced for relay node placement, which minimizes the installation cost and the consumed energy of the network. In [12] the authors introduced a TDMA-based MAC to employ a prediction-based transmission scheme. The coordinator decides the optimal relay allocation according to the channel conditions. In [13], relaying nodes are placed on the body surface to maximize the lifetime of implants, while 
[14] proposes a markovian model to decide transmission. A node switches between relayed and direct transmission, based on the channel quality estimation. The proposed algorithm in [15] uses a relay selection mechanism by considering the received signal strength values of Ack packets from the candidate relaying nodes. The proposed method in [16] is implemented in three parts: the coordinator calculates the channel condition for each pair of nodes, decides the transmission strategy and calculates the transmission power level in each case. Finally, a special note for [17], which is related to the present work: in this paper, the authors introduce a relay mechanism with predefined relays to reduce the failure rate and extend the network lifetime. The protocol was compared to a simplified version of the two-hop extended star topology of IEEE 802.15.6-2012.

\section{B. Motivation and contribution}

The common approach of the aforementioned works is the optimization of the energy consumption. Due to the small size of BANs, such attempts usually result to application specific designs, depending on the assumptions and the specific positions of the nodes. In this paper, we present an effective application-agnostic communication scheme for relaying, using a simple store and forward technique, compatible with IEEE 802.15.6-2012, which allows optimization if needed.

Specifically, in this work, we focus on network discovery and relaying in the two-hop area of BANs. We address the issue of disconnected nodes in BANs, caused by high mobility of body parts and weak signals with a mobility aware relaying scheme. This scheme, which lies exclusively in the data link layer, is fully compatible with IEEE 802.15.6-2012, can be enabled as an enhancement and can be considered as an emergency function to save packets by reaching distressed nodes. A special random access phase is added during the superframe, called emergency phase (EP), after the regular random access phase (RAP1). When EP starts, a rescue beacon is transmitted from every relaying capable node to reach disconnected nodes. A disconnected node may transmit its data packets to its relaying node during EP with CSMA/CA. These packets are buffered and relayed during the next available access phase of the superframe. The proposed scheme is simulated and compared to two-hop star topology extension of the standard with Castalia [18], a framework of OMNeT++ [19].

\section{Outline}

The rest of this paper is organized as follows. In Section II, we present the provided two-hop extension of IEEE 802.15.62012 and introduce the proposed scheme. In Section III, we present in detail the system model for our simulations. In Section IV, there is a discussion on the received results. Finally, Section V concludes the paper.

\section{A New Relaying Scheme}

Relaying in BANs is rarely a design choice based on the topology of the application. In practice, alternative paths are needed to cope with frequent node disconnections caused by

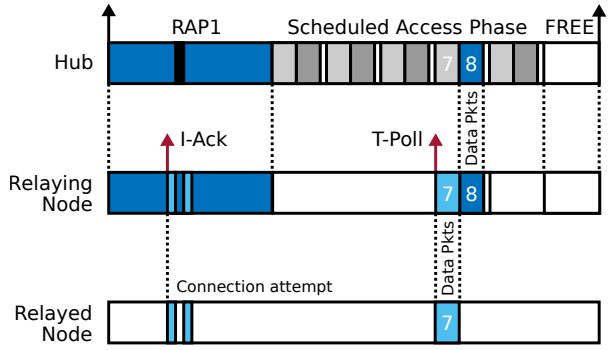

Figure 2. Superframe with two-hop star topology extension enabled

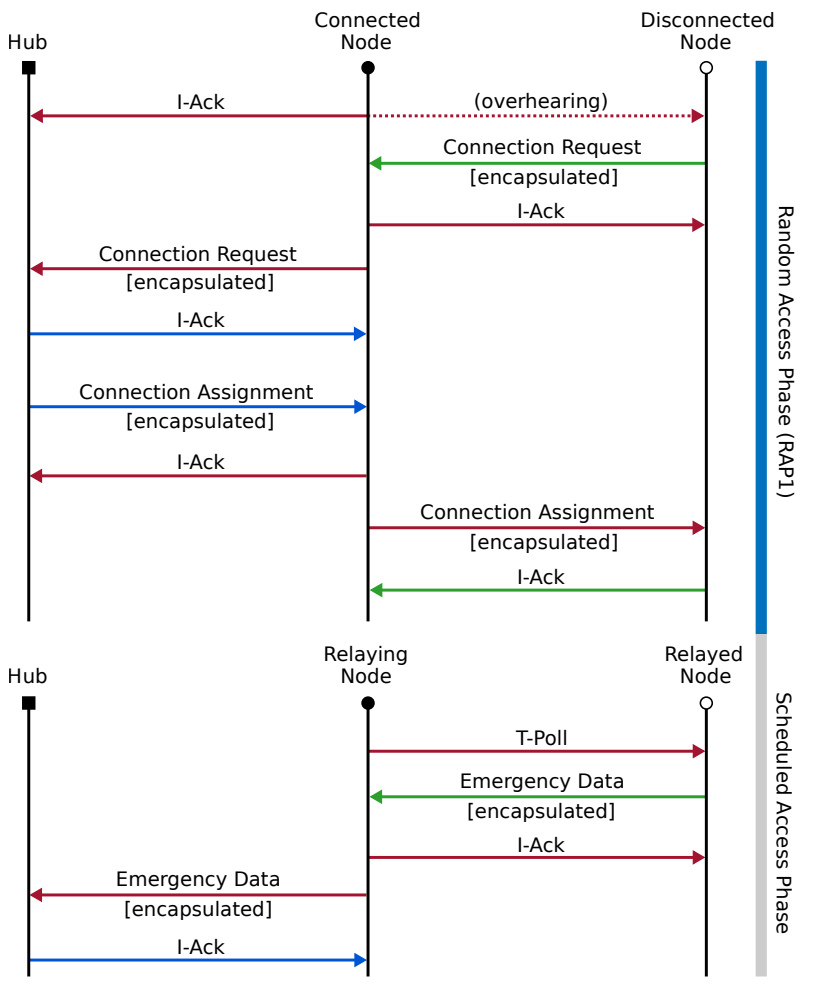

Figure 3. Communication with two-hop star topology extension enabled

low signals and high mobility. Moreover, as it is highlighted in [20], relaying is a desirable function in order to improve reliability. Since multiple hops may introduce large delays, we focus on the two-hop area of the body. This is supported by the standard as it is a reasonable choice for the size of BANs. The admittance of a small amount of delay can be justified by the discovery of more nodes and the reception of more packets. Moreover, relaying has plenty of room for optimization. For example, the selection of a relaying node may depend on its remaining energy or the quality of its connection to the hub denoted by received signal strength indicator (RSSI). We proceed with a description of the twohop star topology extension of IEEE 802.15.6-2012 in II-A, the proposed mobility aware relaying scheme in II-B and a brief discussion on their key differences in II-C.

\section{A. Two-hop star topology extension}

In IEEE 802.15.6-2012, communication takes place between a hub and its connected nodes in star topology. The most common access mode is beacon with superframes. In this 
case, time is divided into beacon periods, also known as superframes. During each superframe the hub may employ random, improvised or scheduled access according to the specific design. There is no doubt that star topology is the most efficient because it provides direct communication between the nodes and the hub, but in many cases it can be insufficient. Since relaying is an important function for BANs, the standard provides a two-hop extension for star topology.

A detailed version of the superframe with two-hop star topology extension it is presented in Fig. 2. This figure shows the discovery mechanism of relaying capable nodes during RAP1 and the relaying procedure during scheduled access phase. The exchanged messages between the nodes during a superframe are depicted in Fig. 3. A disconnected node selects a candidate relaying node by overhearing Ack packets or by receiving a T-Poll frame from a relaying capable node. When a connected node is discovered, it attempts to connect to the hub through that node. The allocation intervals for data packet transmissions from relayed and relaying nodes are assigned by the hub with connection assignments. A relaying node transmits at least one T-Poll frame in each allocation interval for synchronization purposes. A relayed node transmits data packets to its relaying node during a scheduled bilink allocation interval. These data packets are delivered from the relaying node to the hub during a scheduled uplink allocation interval.

\section{B. Mobility aware relaying scheme}

The first results of the proposed scheme were presented in [21]. An early version of this scheme was compared to the baseline standard. It was demonstrated that relaying is feasible and effective with this very simple scheme. Relaying is treated as a special emergency function in addition to the normal operation of the network. We focus on reaching the disconnected nodes, while keeping energy consumption at reasonable levels.

The superframe of the proposed scheme and the exchanged messages between nodes are presented in Figs 4 and 5, respectively. This scheme introduces an additional random access emergency phase (EP) to accelerate relaying process. During EP each connected node to the hub can optionally become a relaying node and send a rescue beacon to reach any possible disconnected node. A disconnected node may accept a rescue beacon from a candidate relaying node, participate in $\mathrm{EP}$ as a relayed node and transmit emergency data packets with CSMA/CA using the contention windows $(\mathrm{CW})$ provided by the standard. The relayed node is not requested to connect to the hub even if it was never seen before. The information about its identity is included in the payload of each data packet. When a relaying node receives a data packet, it responds with an I-Ack and buffers the packet to relay it to the hub during a future access phase. Since a sophisticated caching policy is outside the scope of this work, we use an additional emergency buffer (EM Buffer) for relayed packets, with high priority.

The transmission of a rescue beacon and relay selection can be regarded as optimization problems depending on the application. Fig. 6 demonstrates the procedure of network

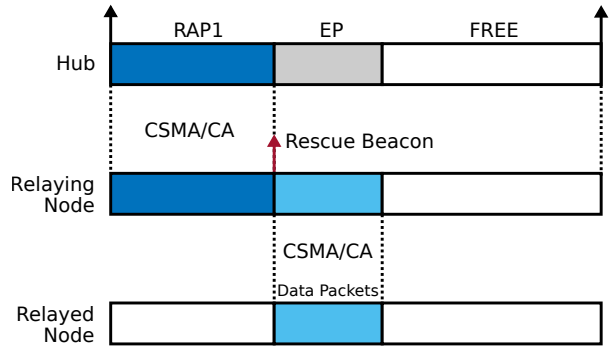

Figure 4. Superframe with mobility aware relaying scheme enabled

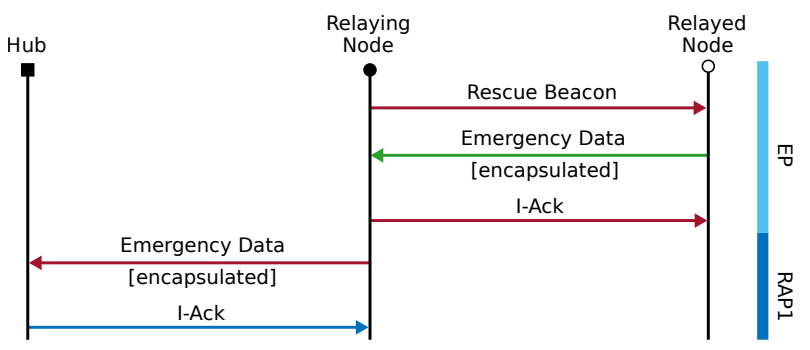

Figure 5. Communication with mobility aware relaying scheme enabled

discovery. A connected node may become a relaying node according to its remaining energy or its link quality with the hub. Similarly, if a disconnected node receives more than one rescue beacons, it may select one of the candidate relaying nodes according to their remaining energy or their link quality.

\section{Pros and cons of the two schemes}

A brief discussion is needed regarding the key differences between the two aforementioned communication schemes, in order to point out their advantages and disadvantages. The operation of each scheme is investigated, by taking into account the implications of mobility.

The two-hop star topology extension is a reasonable choice in terms of energy saving or packet delivery for activities with low mobility, such us sleeping, because the alternative path decided by the hub can be used for long periods of time. In such cases, scheduled access guarantees the safe delivery of relayed packets without collisions.

A couple of issues are related to the connection procedure of a relayed node through a relaying node. In the rapidly changing environment of BANs it is expected that the disconnected nodes attempt to reconnect multiple times. Depending on the timescale and the length of RAP1, the time needed for the connection process can be too much. Moreover, the overhead caused by the repeated connection attempts may have

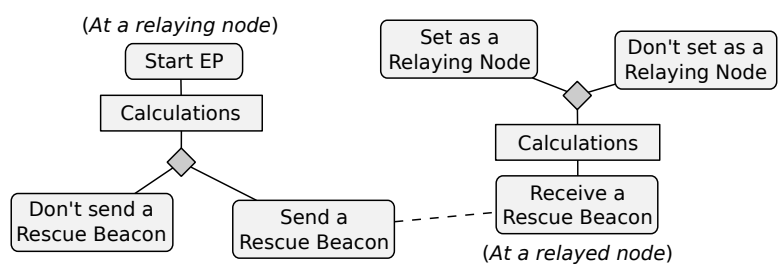

Figure 6. Network discovery with mobility aware relaying scheme enabled 
a negative effect on packet reception, because the connection procedure usually takes place during RAP1.

Several issues of two-hop star topology extension are related to network discovery. The connection process is initiated by overhearing Ack packets sent by relaying nodes to the hub. In practice, this is a rather uncertain way of connecting to the network. It can be observed that the disconnected nodes have very few chances to connect, because the aforementioned Acks are sent to the hub only after the reception of connection assignments. If a node stays connected for a long time, the disconnected nodes do not have any other chances to connect. Also, if a relayed node stays connected for a long time with its relaying node, it keeps transmitting even if its relaying node is disconnected and it misses any opportunity to connect directly to the hub in a future superframe.

The efficiency of this scheme depends mainly on the slot assignment. Relaying and relayed nodes are expected to vary and their future state has to be predicted. Thus, in order to exploit efficiently every slot of the superframe, sophisticated algorithms have to be invented in order to recalculate free space in every superframe and the connections have to be modified accordingly with new connection assignments. This can be very complicated and a huge overhead for BANs. Apparently, if scheduled access is designed for a specific application with low mobility and limited pairs of relayed and relaying nodes, it is possible to get satisfying results by employing a simple TDMA scheme.

The proposed scheme aims to overcome the implications caused by the complex operation of the standard by using connectionless communication and random access. It is a simple and flexible scheme for relaying, which can be used in several cases and eliminates the need of application specific design. It follows an aggressive strategy by employing a periodic transmission of a rescue beacon from each relaying capable node, in order to boost packet transmission from relayed nodes.

With connectionless communication, a relayed node is able to transmit its packets immediately using random access. Relayed nodes are regarded as disconnected and wait for a beacon from the hub to initiate a connection procedure. Thus, the extra burden of frequent reconnections is avoided and relayed nodes are able to connect directly to the hub as soon as possible. However, buffering at relaying nodes suggests that an amount of latency can be tolerated. Latency can be minimized with an additional random access phase (RAP2) or scheduled access after EP for immediate packet delivery to the hub. The expected increase of energy consumption due to random access can be compensated with increased packet delivery.

This scheme can be easily adapted to more than two hops to serve hierarchical structures and reach more distant nodes using additional rescue beacons. Due to the small size of BANs, the usefulness of multi-hop access is questionable, but it can be investigated in the future.

The proposed scheme operates using the communication methods described in the standard and provides some opportunities for optimization if required by the application. Overall, it has an important advantage due to its simple implementation: it runs effectively out of the box, without any application

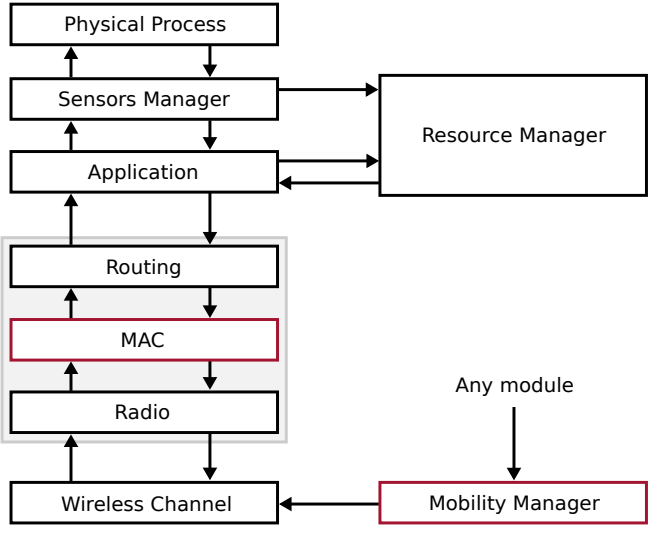

Figure 7. The structure of Castalia; our contribution is deployed as MAC and mobility modules

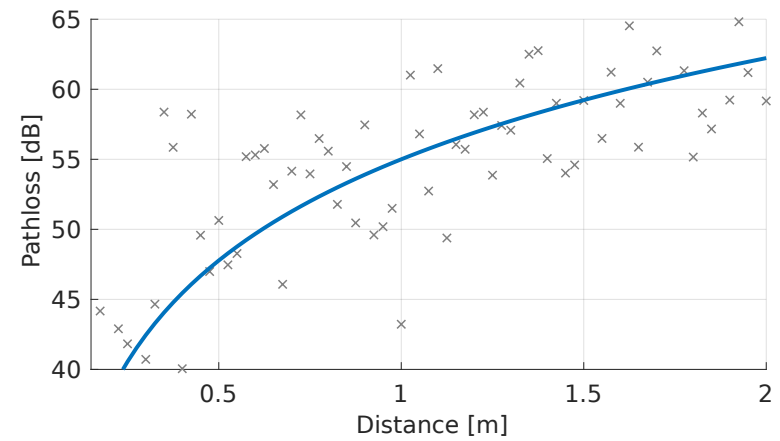

Figure 8. Pathloss in $2.4 \mathrm{GHz}$ (Castalia)

specific configuration.

\section{System AND Channel Model}

The system model follows the structure of Castalia, as in Fig. 7. New MAC and mobility modules were developed for the additional functionality. Every other module has the default values of Castalia, in order to provide reproducible results and facilitate any future comparisons with other MAC schemes. Randomness is employed at several cases, such as the transmission times, the switching between $\mathrm{Rx}$ and $\mathrm{Tx}$ states and the calculation of pathloss. Energy consumption is calculated by the Resource Manager. The rest of this section includes the most important choices regarding the channel in III-A, mobility in III-B and MAC in III-C.

\section{A. Channel model}

The quality of a signal around a human body is subject to the typical causes of degradation: fading, pathloss and shadowing. Pathloss is modeled as a function of distance between two nodes for several frequencies and scenarios depending on node placement. The analytic and measurements work carried out over the years is included in [22], the official technical report for IEEE P802.15.

In this work, we use the band of $2.4 \mathrm{GHz}$ for reference purposes as it is a widely used frequency in Wireless Sensor Networks (WSNs) and Wireless Local Area Networks 


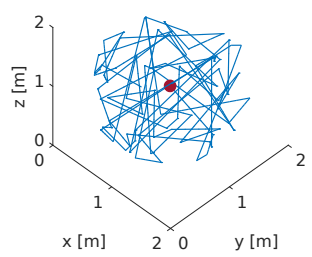

(a) Random walk mobility

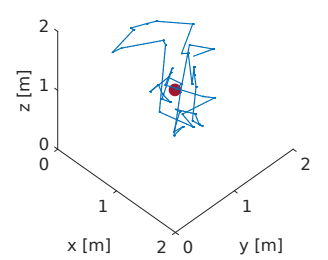

(b) Random waypoint mobility

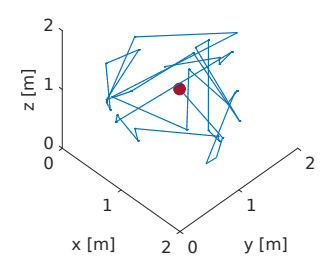

(c) Random direction mobility

Figure 9. Traces of several stochastic mobility models for a single node

(WLANs) as well. The default pathloss model in $2.4 \mathrm{GHz}$ from Castalia is

$$
P L(d)_{\mathrm{dB}}=P L\left(d_{0}\right)+10 \eta \log _{10}\left(d / d_{0}\right)+X\left(\mu, \sigma^{2}\right),
$$

where $P L\left(d_{0}\right)=55 \mathrm{dBm}$ is the pathloss at reference distance $d_{0}=1 \mathrm{~m}, \eta=2.4$ is the pathloss exponent indicating the speed of signal strength fading and $d$ is the distance between two nodes. $X$ is a normally distributed variable with $\mu=0$ and $\sigma=4$. According to Fig. 8, high values for pathloss are expected, even for short distances.

In Castalia, 3D space is divided in small cells and pathloss is calculated from/to each other cell. This results to a very fast execution of the simulation but it is very demanding in memory; the smaller the cell gets the larger amount of memory is needed. This is one of the trade-offs, the cell size has to be selected according to the desired accuracy and memory availability. In our simulations, each cell's side size is $10 \mathrm{~cm}$. While this channel modeling method lacks in accuracy, it gives valuable insight.

\section{B. Mobility model}

Mobility models are usually categorized as entity or group models [23]. Entity modeling refers to the movement patterns of a single node. Group modeling suggests that the nodes are in some way related and move around a common logical center. Specifically, in the context of BANs, entity and group models are expected to simulate the movement of body parts. In this work, we choose a higher level of abstraction and categorize mobility models as stochastic or trace based.

1) Stochastic mobility: A stochastic model implies that each node moves randomly inside a region. This movement can be computed using one of the widely used mobility models: random walk mobility (RWM), random waypoint mobility (RWP) and random direction mobility (RDM), as described in [24]. Furthermore, there are stochastic models for a group of nodes in the area of the human body. The most notable is reference point group mobility (RPGM) [24] and MoBAN [25] as an extension of it. RPGM uses a logical center

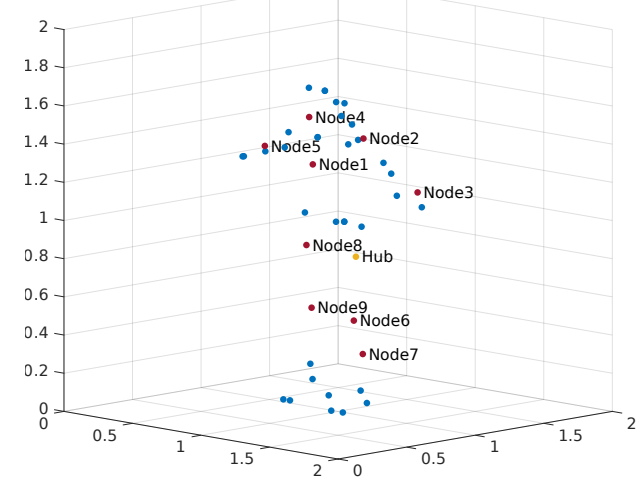

Figure 10. A snapshot of a trace based mobility model for a group of nodes

for a group of nodes with RWM and MoBAN introduces a Markov chain with the transition probabilities between several human postures. To test stochastic mobility we developed RWM, RWP and RDM models for Castalia. Each model describes the movement of a single node inside a sphere. A node with RWM or RDM travels to random directions and a node with RWP travels from point to point. Four stochastic parameters are utilized: (i) the radius of the sphere, (ii) the speed, (iii) the travel time for the movement in each direction (RWM) and (iv) the pause time at each destination (RWP, RDM). Except for the radius, every parameter is selected uniformly on the provided intervals. A visual representation of the three models can be observed in Fig. 9. The movement of a node according to these models is unrealistic. Moreover, if we consider that the movement of a single node is usually correlated to the position of another node (e.g. a node on the hand and a node on the arm) then it seems impossible to model human mobility using random mobility models.

2) Trace based mobility: Specifically in BANs, the nodes are related in a way that they cannot be treated independently, so it is not feasible to use random mobility models for single nodes. In such cases, the way to implement mobility accurately is to employ data of real traces of human body parts. Trace based mobility is possible due to the fact that there is already a great amount of motion capture data available online from unrelated scientific fields such as image processing and gait analysis. The available datasets represent several everyday activities. These data are usually captured with commercially available devices and they are stored in several formats such as $\mathrm{BVH}, \mathrm{AMC}$ and $\mathrm{C} 3 \mathrm{D}$ [26], depending on the application. In this work, we use C3D data because they can be easily imported and processed in several programming environments. Specifically, the selected dataset is imported in Matlab, the nodes are placed at the desired positions and the traces are exported in comma separated value (CSV) format using a custom script. The deployed mobility module in Castalia parses these data and updates the position of each node during the simulation. The data points are usually provided in $120 \mathrm{fps}$, so the traces are selected according to the refresh rate of the simulation. In Fig. 10 there is a visual representation of one of the most used datasets in our simulations, from Mocap Database HDM05 [27], representing a man practicing rope 


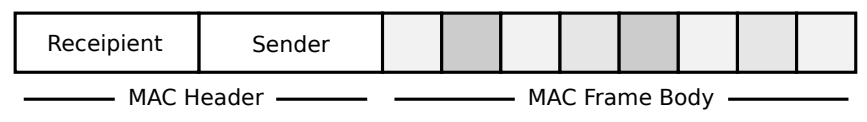

Figure 11. A simplified MAC frame structure

skipping. A video of this model can be watched in [28].

\section{C. $M A C$}

In order to demonstrate relaying we created a new relaying capable MAC module based on Baseline MAC [18] which is the baseline implementation of IEEE 802.15.6-2012 by the creators of Castalia. MAC frames are structured as in Fig. 11. The implemented MAC module sets the Radio to TX, RX or SLEEP and coordinates random and scheduled access. It supports the basic functions of the standard and introduces the two aforementioned relaying schemes: twohop star topology extension of the standard and the proposed mobility aware relaying scheme. To initiate a relaying process we need to know if a node is connected or not. In this work, a node disconnects if it does not receive a beacon in $0.5 \mathrm{~s}$. In the following paragraphs the discussion is focused on design choices related to the implementations of the two schemes.

1) Two-hop star topology extension: Upon a successful connection, the hub implements a simple TDMA scheduling protocol by assigning the same number of slots for each relayed node and its relaying. For example, if two slots are assigned for the relayed and two slots for its relaying node, six pairs of nodes can be accommodated during 24 free slots of a superframe. The hub attempts to reuse these slots as often as possible by checking if a pair of nodes was seen before. Inevitably, if relayed nodes are too many, the superframe runs out of space and new connection requests are declined. In fact, as it was previously hinted, this is the major weakness of this scheme. It seems impossible to employ a fair scheduling for every set of relayed nodes without any optimization.

2) Mobility aware relaying scheme: This scheme provides the opportunity to disconnected nodes to transmit their packets in every superframe. Each connected node becomes a relaying node and transmits a rescue beacon at the beginning of EP. A relaying node waits in Rx mode during EP to receive data packets from relayed nodes. A disconnected node accepts the first rescue beacon it receives and becomes a relayed node. Then, it competes for channel access with CSMA/CA, using the contention windows $(\mathrm{CW})$ specified in the standard. Each relayed node selects a random time interval to attempt a future transmission inside a contention window. The received packets by relayed nodes are placed in a special buffer with high priority (EM Buffer) for future transmission. Whenever there is an opportunity for transmission, EM Buffer is checked first for any remaining packets.

\section{Simulation And Results}

The simulations run, while the human subject practices rope skipping. The node configuration of Fig. 12 includes the most common positions for sensing. Nine nodes are placed on body

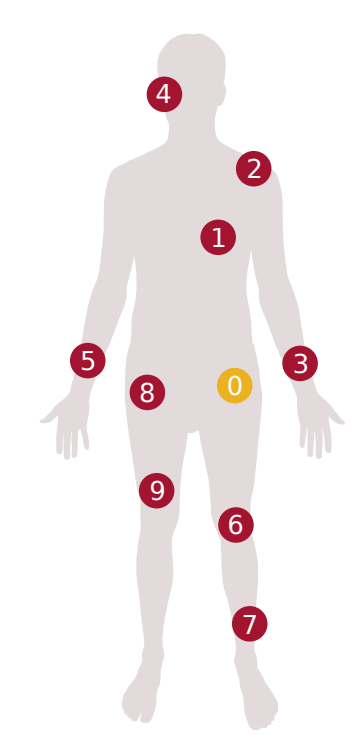

\begin{tabular}{l|l}
\hline Parameter & Value \\
\hline \hline Time & $60 \mathrm{~s}$ \\
\hline Nodes & $9+$ Hub \\
\hline Payload & 100 bytes \\
\hline Packet rate & 20 packets $/ \mathrm{s}$ \\
\hline \hline Mobility & Rope skipping [28] \\
\hline \hline Allocation slot & $10 \mathrm{~m} \mathrm{sec}$ \\
\hline Superframe & 32 slots \\
\hline RAP1 & 8 slots \\
\hline EP & 4 slots \\
\hline Scheduled access & 2 slots (each node) \\
\hline MAC Buffer & 16 frames \\
\hline EM Buffer & 16 frames \\
\hline \hline Carrier frequency & $2.4 \mathrm{GHz}$ \\
\hline Modulation & DQPSK \\
\hline TX power & $-15 \mathrm{dBm}$ \\
\hline RX sensitivity & $-87 \mathrm{dBm}$ \\
\hline \hline Channel & Lognormal (Castalia) \\
\hline
\end{tabular}

Figure 12. Simulation setup: Node placement and Parameters

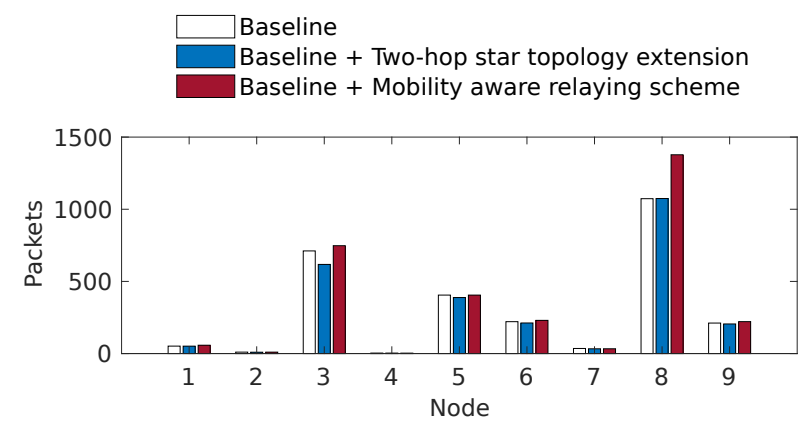

Figure 13. Directly received data packets at the hub

surface and send data packets to the hub. This simulation is repeated 100 times using a different seed for each run, in order to receive random but reproducible results. The used metrics are the received data packets from each node, the discovered relaying capable nodes and the consumed energy per bit.

Fig. 13 shows the average values of directly received data packets at the hub from each node. The proposed scheme achieves a small improvement compared to the baseline standard and its two-hop star topology extension. The additional packets come from relayed nodes and most of them are delivered by node 8 . In the case of node 3 , the connection attempts of two-hop star topology extension during RAP1 have a negative impact on packet reception.

The aforementioned results depend primarily on network discovery. Fig. 14 validates that the aggressive strategy of the mobility aware relaying scheme to reach disconnected nodes is effective. The improvement of node discovery indicates that disconnected nodes have more opportunities to transmit their data packets to relaying capable nodes. Subsequently, a closer inspection is performed, in order to check which packets are relayed successfully to the hub.

In Fig. 15, the two-hop star topology extension delivers zero or very few packets from each relayed node to the hub. A 


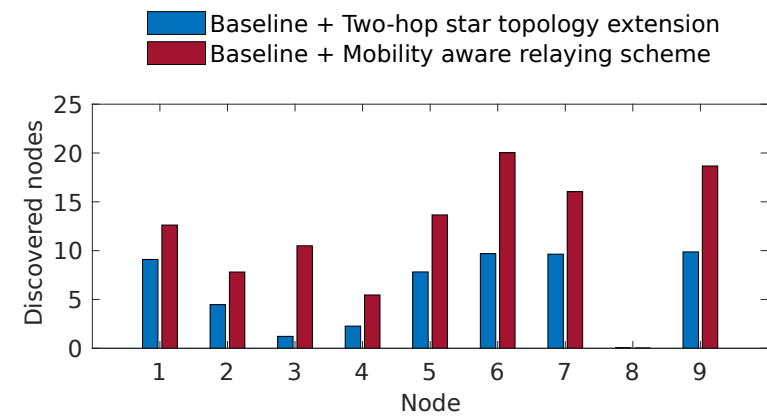

Figure 14. Discovered relaying capable nodes by disconnected nodes

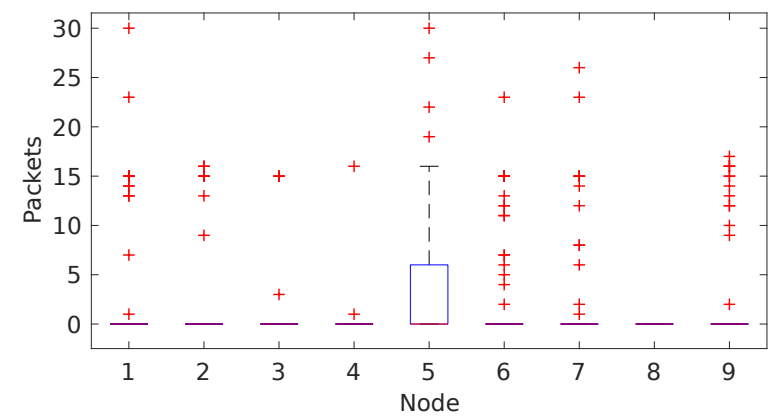

Figure 15. Received data packets at the hub from relayed nodes with two-hop star topology extension enabled

couple of clarifications are needed to explain this performance. As it was already mentioned, the initialization of relaying process is rather uncertain because it depends on overhearing of Acks. If a disconnected node does not receive any Acks, then it does not attempt to connect. Also, in order to be fair to every node, the hub assigns two slots for each relayed and two slots for its relaying node. This policy limits the performance of some of the disconnected nodes which are probably able to deliver more packets.

In Fig. 16, it is evident that the proposed scheme delivers many more packets from relayed nodes to the hub by occupying only 4 slots in each superframe for random access. The strong advantage is the immediate transmission of data packets after the discovery of a connected node. Moreover, the outliers indicate that there are bursts of traffic during some

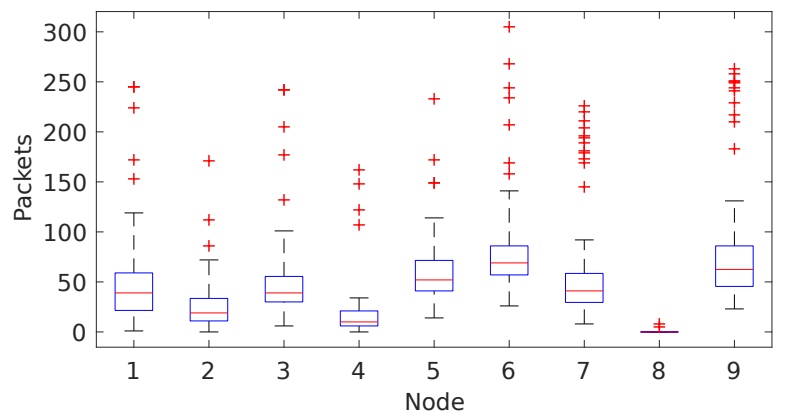

Figure 16. Received data packets at the hub from relayed nodes with mobility aware relaying scheme enabled

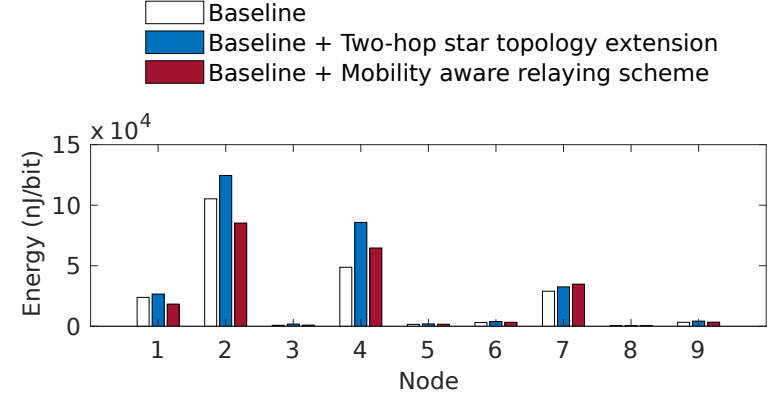

Figure 17. Consumed energy per bit (nJ/bit)

realizations. In practice, mobility can either assist or prevent packet delivery.

In Fig. 17, energy per bit is the consumed energy at each node divided by the number of successfully transmitted bits. It is expected that random access consumes more energy compared to scheduled access, due to collisions. However, the proposed scheme has marginally better results because energy consumption depends primarily on idle listening. Thus, an increased number of successfully transmitted packets has a strong impact on energy per bit ratio. The fact that the results of the two schemes are comparable shows that the additional energy consumption due to random access is acceptable.

To sum up, simulation results show that the proposed scheme has a better performance in terms of node discovery, packet delivery and energy per bit, because it is a general purpose, mobility aware relaying scheme, based on random access. On the other side, the two-hop star topology extension of the standard has to be tuned for a specific application and node placement, preferably with low or no mobility, in order to get the most out of it.

\section{CONCLUSION}

Relaying is a useful feature for BANs and is necessary for the devices with the current sensitivity and weak signals. The two-hop star topology extension of the standard employs scheduled access, which may become insufficient in cases of high mobility due to the unpredictable states of the disconnected nodes. The proposed scheme is simple, flexible and operates according to the standard by employing a short emergency random access phase dedicated for disconnected nodes. A possible increase in energy consumption due to random access is compensated with increased packet delivery. Our implementation in Castalia will be available to serve as a testbed for further research.

\section{REFERENCES}

[1] IEEE Standard for Local and metropolitan area networks - Part 15.6: Wireless Body Area Networks, IEEE Std. 802.15.6, Feb. 2012.

[2] G. Fortino, R. Giannantonio, R. Gravina, P. Kuryloski, and R. Jafari, "Enabling effective programming and flexible management of efficient body sensor network applications," IEEE Transactions on HumanMachine Systems, vol. 43, no. 1, pp. 115-133, Jan. 2013.

[3] G. Smart, N. Deligiannis, R. Surace, V. Loscri, G. Fortino, and Y. Andreopoulos, "Decentralized time-synchronized channel swapping for ad hoc wireless networks," IEEE Transactions on Vehicular Technology, vol. 65, no. 10, pp. 8538-8553, Oct. 2016. 
[4] Smart Body Area Network (SmartBAN); Low Complexity Medium Access Control (MAC) for SmartBAN, ETSI Working Draft Proposed Standard, Rev. DTS/SmartBAN-005, Apr. 2015.

[5] B. Latre, B. Braem, I. Moerman, C. Blondia, E. Reusens, W. Joseph, and P. Demeester, "A low-delay protocol for multihop wireless body area networks," in 2007 Fourth Annual International Conference on Mobile and Ubiquitous Systems: Networking Services (MobiQuitous), Philadelphia, PA, USA, Aug. 2007, pp. 1-8.

[6] M. Maman and L. Ouvry, "Batmac: An adaptive tdma mac for body area networks performed with a space-time dependent channel model," in 2011 5th International Symposium on Medical Information and Communication Technology, Montreux, Switzerland, Mar. 2011, pp. 1-5.

[7] M. Maman, D. Miras, and L. Ouvry, "Implementation of a selforganizing, adaptive, flexible and ultra low-power mac protocol for wireless body area networks," in 2013 IEEE 24th Annual International Symposium on Personal, Indoor, and Mobile Radio Communications (PIMRC), London, UK, Sep. 2013, pp. 1737-1742.

[8] M. Nabi, T. Basten, M. Geilen, M. Blagojevic, and T. Hendriks, "A robust protocol stack for multi-hop wireless body area networks with transmit power adaptation," in Proceedings of the Fifth International Conference on Body Area Networks, Corfu, Greece, Sep. 2010, pp. $77-$ 83.

[9] M. Nabi, M. Geilen, and T. Basten, "On-demand data forwarding for automatic adaptation of data propagation in wbans," in 2012 9th Annual IEEE Communications Society Conference on Sensor, Mesh and Ad Hoc Communications and Networks (SECON), Seoul, South Korea, Jun. 2012, pp. 326-334.

[10] C. Lin and P. Chuang, "Energy-efficient transmission in wireless body area networks," in 2012 International Symposium on Intelligent Signal Processing and Communications Systems, Taipei, Taiwan, Nov. 2012, pp. 663-668.

[11] J. Elias, A. Jarray, J. Salazar, A. Karmouch, and A. Mehaoua, "A reliable design of wireless body area networks," in 2013 IEEE Global Communications Conference (GLOBECOM), Atlanta, GA, USA, Dec. 2013, pp. 2742-2748.

[12] H. Feng, B. Liu, Z. Yan, C. Zhang, and C. W. Chen, "Prediction-based dynamic relay transmission scheme for wireless body area networks," in 2013 IEEE 24th Annual International Symposium on Personal, Indoor, and Mobile Radio Communications (PIMRC), London, UK, Sep. 2013, pp. 2539-2544.

[13] N. Javaid, A. Ahmad, Y. Khan, Z. A. Khan, and T. A. Alghamdi, "A relay based routing protocol for wireless in-body sensor networks," Wireless Personal Communications, vol. 80, no. 3, pp. 1063-1078, Feb. 2015.

[14] A. Maskooki, C. B. Soh, E. Gunawan, and K. S. Low, "Adaptive routing for dynamic on-body wireless sensor networks," IEEE Journal of Biomedical and Health Informatics, vol. 19, no. 2, pp. 549-558, Mar. 2015.

[15] S.-K. Kim and Y.-I. Joo, "A distributed relay selection algorithm for two-hop wireless body area networks," Journal of the Korean Society of Marine Engineering, vol. 41, no. 2, pp. 156-162, Feb. 2017.

[16] Y. Zhang and B. Zhang, "A relay-aided transmission power control method in wireless body area networks," IEEE Access, vol. 5, pp. 84088418, Jun. 2017.

[17] R. Pan, D. Chua, J. S. Pathmasuntharam, and Y. P. Xu, "An opportunistic relay protocol with dynamic scheduling in wireless body area sensor network," IEEE Sensors Journal, vol. 15, no. 7, pp. 3743-3750, Jul. 2015.

[18] Castalia. [Online]. Available: https://github.com/boulis/Castalia/

[19] Omnet++. [Online]. Available: https://www.omnetpp.org/

[20] A. Boulis, D. Smith, D. Miniutti, L. Libman, and Y. Tselishchev, "Challenges in body area networks for healthcare: the mac," IEEE Communications Magazine, vol. 50, no. 5, pp. 100-106, May 2012.

[21] C. Michaelides, M. Iloridou, and F. Pavlidou, "In search of the lost nodes in bans," in 2018 IEEE 26th International Conference on Network Protocols (ICNP), Cambridge, UK, Sep. 2018, pp. 249-250.

[22] K. Y. Yazdandoost and K. Sayrafian-Pour, "Channel model for body area network (ban)," IEEE, Tech. Rep. 15-08-0780-11-0006, Nov. 2010.

[23] T. Camp, J. Boleng, and V. Davies, "A survey of mobility models for ad hoc network research," Wireless communications and mobile computing, vol. 2, no. 5, pp. 483-502, Sep. 2002.

[24] R. R. Roy, Handbook of Mobile Ad Hoc Networks for Mobility Models. Springer, 2011.

[25] M. Nabi, M. Geilen, and T. Basten, "Moban: A configurable mobility model for wireless body area networks," in Proceedings of the 4th International ICST Conference on Simulation Tools and Techniques, Barcelona, Spain, Mar. 2011, pp. 168-177.
[26] The 3d biomechanics data standard. [Online]. Available: https: //www.c3d.org/

[27] M. Müller, T. Röder, M. Clausen, B. Eberhardt, B. Krüger, and A. Weber, "Documentation mocap database hdm05," Universität Bonn, Tech. Rep. CG-2007-2, Jun. 2007.

[28] Rope skipping. [Online]. Available: https://youtu.be/yDXF1JRYPSo

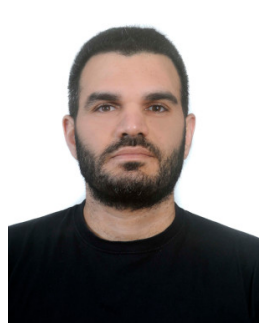

Costas Michaelides studies Electrical and Computer Engineering at Aristotle University of Thessaloniki, Greece. His research interests include the design and performance evaluation of Sensor and Body Area Networks.

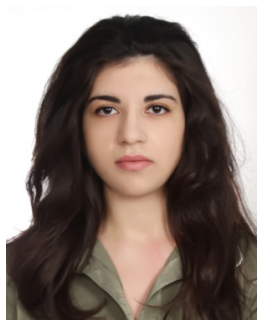

Maria Iloridou received the Diploma degree in Electrical and Computer Engineering from the Aristotle University of Thessaloniki, Greece in 2012. She is currently a $\mathrm{PhD}$ candidate in the same department, where she also serves as a teaching assistant. Her research interests include MAC protocol analysis in $4 \mathrm{G}$ networks and clustering in wireless mobile sensor networks.

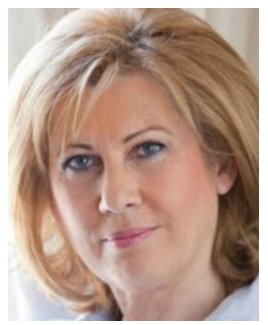

Fotini-Niovi Pavlidou Mechanical/Electrical Engineering degree and $\mathrm{PhD}$ degree from Aristotle University of Thessaloniki (AUTh), Greece, is currently a full professor at the department of Electrical and Computer Engineering (ECE) in AUTh. She is engaged in research and lectures in the fields of Mobile Communications, Internet of Things, Satellite Communications, Sensor and Body area networks. Her research interests include traffic analysis, protocol design, performance evaluation and QoS services for multimedia applications. She is the author of more than 150 papers in international journals and conferences. She has been involved in various IEEE editorial activities and has been the organizer and Technical Program Chair of a number of IEEE conferences. Prof. Pavlidou has been involved in many European and National Projects. She has been the Delegate of Greece in the European COST Program on Telecommunications (1998-2004), has served as an Expert for Greece in the FP7 ICT Cooperation Program (2007-2010), as a representative of Greece in the Public Authorities Board (PAB) of the ARTEMIS Joint Undertaking (2008-2010) and as a national delegate for the FP7 Capacities Program on SMEs. She is a Senior Member of IEEE, has served as chairperson for the Joint VTS \& AESS Greece Chapter (1999-2012), as Officer for Continuing Education (CE) IEEE Region 8 (2011-2013), as Coordinator of Educational Activities Subcommittee (EASC) Region 8 (2013-2015) and as a member of the IEEE Section Outreach Committee (SEOC) of the Educational Activities Board (EAB) (2013-2015). http://newton.ee.auth.gr/pavlidou/ 